\title{
Zero-Time One-Particle States in Quantum Field Theory
}

\author{
Etienne Frochaux \\ Département de Mathématiques, Ecole Polytechnique Fédérale de Lausanne, \\ $\mathrm{CH}-1015$ Lausanne, Switzerland
}

Received January 2, 1990; in revised form June 15, 1990

\begin{abstract}
In the framework of the weakly-coupled $P(\varphi)_{2}$-models we construct perturbation approximations of vectors of a dense set of the state space, especially vectors of the one-particle state subspace, by polynomials of zero-time fields acting on the vacuum state, with rigorous control of the remainders.
\end{abstract}

\section{Introduction}

Motivation. The particle structure of a Quantum Field Theory model is generally deduced from the analyticity properties of the Green distributions, obtained from the study of the Bethe-Salpeter equation. This gives precise information about the spectrum of $M$, the mass operator (see the references in [2], to which we must add now [1]). Another method, not completely independent, is the variational perturbation method, intially proposed by Glimm, Jaffe and Spencer [8], and studied in [2] for same $P(\varphi)_{2}$-models. For all $f \in L^{1} \cap L^{2}\left(\mathbb{R}^{2}\right), f \neq 0$, a vector $\Psi(f)$ is constructed, which satisfies the following conditions:

1. it is a linear combination of zero-time fields acting on the vacuum, 2 . it is orthogonal to the vacuum and to the one-particle states, 3. it lies in the domain of $M$, and 4 . it verifies the following formula:

$$
\frac{\left(\Psi(f) ; M^{2} \Psi(f)\right)}{(\Psi(f) ; \Psi(f))}=(2 m)^{2}+4 \frac{\lambda^{2}}{m} \frac{\left\langle f ; H_{\mathrm{rel}}^{\mathrm{NR}} f\right\rangle}{\langle f ; f\rangle}+O\left(\lambda^{5 / 2}\right)
$$

for $\lambda$, the coupling constant, sufficiently small. Here $(\because)$ is the state-space scalar product, $m$ is the one-particle physical mass, $\langle\because\rangle$ is the $L^{2}\left(\mathbb{R}^{2}\right)$ scalar product and $H_{\mathrm{rel}}^{\mathrm{NR}}$ is the relative Hamiltonian of the Non-Relativistic limit, written for $\lambda=m^{2}$. A careful study of the remainder, which is $O\left(\lambda^{5 / 2}\right)$, has been made. The above formula has been established for all $P(\varphi)_{2}$-models with even interaction polynomial $P$ having a non-zero fourth degree term. For a discussion on the conclusions that can be drawn apropos of the spectrum of $M$ and its eigenvectors, see [2]. 
The generalization for all $P(\varphi)_{2}$-models with weak coupling [3] and the calculation of the higher perturbation terms [4] require the knowledge of one-particle states given by polynomials of zero-time fields acting on the vacuum state, and of some products of such states.

Contents. This work is concerned with the zero-time fields acting on the vacuum state (we call them zero-time vectors) for all weakly-coupled $P(\varphi)_{2}$-models. We show in Sect. 1 that all vectors given by a product of fields acting on the vacuum state can be approached perturbatively by a linear combination of zero-time vectors. With Theorem 3 we see that the series are asymptotic. Thus for all perturbation calculations, it is enough to consider only zero-time vectors. In Sect. 2, we expand $\left(M^{2}-z\right)^{-1} \xi$, for $z \in \mathbb{C}$ and $\xi$ a zero-time vector, as a perturbation combination of zero-time vectors, and in Theorem 5 we show that the series is asymptotic. In Sect. 3, we construct approximations of one-particle states at zero-time, and collect their properties. Section 4 introduces vectors obtained by some tensorial products of such states, which will give good ansatz to approach the $n$-particle states at low energy.

Notation. The weakly-coupled $P(\varphi)_{2}$-models are defined by their Schwinger distributions $S_{n}$, given by the moments of a probability space $(Q, \Sigma, \mu)$, where $Q=\mathscr{S}^{\prime}\left(\mathbb{R}^{2}\right)$ (real valued), $\Sigma$ is the Borel $\sigma$-algebra of $Q$ (given the weak topology), and $\mu$ is a probability measure on $\Sigma$. For all $f \in \mathscr{S}\left(\mathbb{R}^{2}\right)$ we introduce $\phi(f)$, the Euclidean fields, defined by $\phi(f)(q)=q(f)$ for all $q \in Q$. Then the Schwinger distributions are given by: $S_{n}(f)=\int_{Q} \phi\left(f_{1}\right) \cdots \phi\left(f_{n}\right) d \mu$ for all $f \in\left(\mathscr{S}\left(\mathbb{R}^{2}\right)\right)^{n}$. The measure $\mu$ is constructed as a double limit: $\mu(B)=\lim _{\Lambda \rightarrow \mathbb{R}^{2}} \lim _{g \rightarrow \delta} \mu_{g}, \Lambda(B)$ for all $B \in \Sigma$, where for all $\Lambda$ compact set of $\mathbb{R}^{2}, g \in L^{2}\left(\mathbb{R}^{2}\right)$ and $q \in \overrightarrow{\mathbb{Q}}$ :

$$
d \mu_{g, \Lambda}(q)=\left(Z_{g}, \Lambda\right)^{-1} d \mu_{0}(q) \exp \left(-\lambda \int_{\Lambda} d^{2} x: P(\phi(x \cdot g)(q)):\right)
$$

where $Z_{g, \Delta}$ is the normalization factor and $\mu_{0}$ is the probability measure on $\Sigma$ such that $\int_{Q} d \mu_{0} \exp i \phi(f)=\exp -\frac{1}{2}\left\langle f,\left(-\Delta+m_{0}{ }^{2}\right)^{-1} f\right\rangle$ for all $f \in \mathscr{S}\left(\mathbb{R}^{2}\right) . P$, the interaction polynomial, is an $\mathbb{R} \rightarrow \mathbb{R}$ polynomial bounded from below, $\lambda \geqq 0$ is the coupling constant, $m_{0}>0$ is the one-particle mass of the so-called free theory, the theory in which $\lambda=0$. :.: denotes the Wick polynomial procedure. There exists $\lambda>0$, depending on $m_{0}$ and $P$, such that the double limit exist if $\lambda \in[0, \lambda]$, which will be assumed throughout this paper. The Schwinger distributions satisfy the Osterwalder-Schrader axioms, thus they define a Wightman Quantum Field model, with state space $\mathscr{H}$, Hamiltonian $H$, momentum $\vec{P}$, vacuum $\Omega$ and field $\varphi$. We denote by $W$ the canonical map $L^{2}(Q, \mu) \rightarrow \mathscr{H}$. For more details, references and other notations (such as for the Fourier transformation), see [5].

Background. For $n \in \mathbb{N}^{*}$, let $\theta^{n}(f, \stackrel{\circ}{x})$ be the Wick-monomial of Euclidean fields with fixed Euclidean times given by:

$$
\theta^{n}(f, \stackrel{\circ}{x})=\int_{\mathbb{R}^{n}} d^{n} \vec{x} f(\vec{x}): \phi\left(\stackrel{\circ}{x}_{1}, \vec{x}_{1}\right) \cdots \phi\left(\stackrel{\circ}{x}_{n}, \vec{x}_{n}\right):
$$

for all $\stackrel{\circ}{x} \in \mathbb{R}^{n}$ and $f \in \mathscr{S}\left(\mathbb{R}^{n}\right)$. Such objects can be defined as strong limits in $L^{2}(Q, \mu)$; here $\phi(x)$ stands for $\phi(x \cdot \delta)$. Let $\mathscr{B}_{n}$ and $\mathscr{B}_{n}^{\alpha}$ for all $\alpha \in \mathbb{N}$ be the spaces of generalized 
functions $f \in \mathscr{S}^{\prime}\left(\mathbb{R}^{n}\right)$ with continuous Fourier transform $\tilde{f} \in C^{0}\left(\mathbb{R}^{n}\right)$ and with bounded semi-norm $b_{n}(f)$ and norm $b_{n}^{\alpha}(f)$ respectively, given by:

$$
\begin{aligned}
& \left(b_{n}(f)\right)^{2}=\int_{\mathbb{R}^{n}}\left(\prod_{j=1}^{n} \frac{d \vec{k}_{j}}{\omega_{0}\left(\vec{k}_{j}\right)}\right)|\tilde{f}(\vec{k})|^{2} \sum_{p \in \mathscr{P}_{n}} \prod_{J \in p} \delta\left(\sum_{j \in J} \vec{k}_{j}\right), \\
& \left(b_{1}^{\alpha}(f)\right)^{2}=\int_{\mathbb{R}} \frac{d \vec{k}}{\omega_{0}(\vec{k})}|\tilde{f}(\vec{k})|^{2}\left(\omega_{0}(\vec{k})\right)^{\alpha}
\end{aligned}
$$

and for $n>1$ :

$$
\left(\ell_{n}^{\alpha}(f)\right)^{2}=\sum_{i=1}^{n-1} \int_{\mathbb{R}^{n}}\left(\prod_{j=1}^{n} \frac{d \vec{k}_{j}}{\omega_{0}\left(\vec{k}_{j}\right)}\right)\left|\tilde{f}_{S}(\vec{k})\right|^{2}\left(\sum_{j=i+1}^{n} \omega_{0}\left(\vec{k}_{j}\right)\right)^{\alpha} \sum_{p \in \mathscr{P}_{i}} \prod_{J \in p} \delta\left(\sum_{j \in J} \vec{k}_{j}\right),
$$

where $\omega_{0}(p)=\sqrt{p^{2}+m_{0}^{2}}$ for all $p \in \mathbb{R}$ and $\mathscr{P}_{i}$ is the set of all partitions of $\{1, \ldots, i\}$. $f_{S}$ is the function obtained from $f$ by symmetrization. It is shown in [6] that $\theta^{n}(f, \dot{x})$ can be defined for $f \in \mathscr{B}_{n}$ and $\mathscr{B}_{n}^{0}$, as strong limits in $L^{2}(Q, \mu)$, and that the vectors $W \theta^{n}(f, \stackrel{\circ}{x})$ and their scalar products satisfy the following theorem. We use the notation $\mathbb{R}_{+}=[0, \infty)$ and $E_{0}$ is the orthogonal projector of $\mathscr{H}$ on $\Omega$.

Theorem 1. For all $\lambda \in[0, \underline{\lambda}], n, m \in \mathbb{N}^{*}, \stackrel{\circ}{x} \in\left(\mathbb{R}_{+}\right)^{n}, \stackrel{\circ}{y} \in\left(\mathbb{R}_{+}\right)^{m}$ :

i) For all $f \in \mathscr{B}_{n}, E_{0} W \theta^{n}(f, \stackrel{\circ}{x})$ is well defined, $\left(W \theta^{n}(f, \stackrel{\circ}{x}) ; \Omega\right)=\int_{Q} d \mu \theta^{n}(f, \stackrel{\circ}{x})$ which is a $C^{\infty}$ function of $\lambda$, and for all $v \in \mathbb{N},\left|\partial_{\lambda}{ }^{v}\left(W \theta^{n}(f, \stackrel{x}{x}) ; \Omega\right)\right|<K b_{n}(f)$ for some $K \in(0, \infty)$ independent of $\lambda, f$ and $\stackrel{\circ}{x}$.

ii) For all $f \in \mathscr{B}_{n}^{0},\left(1-E_{0}\right) W \theta^{n}(f, \stackrel{\circ}{x}) \in\left(1-E_{0}\right) \mathscr{H}$.

iii) For all $\beta \in \mathbb{N}^{*}, \vec{P}^{\beta} W \theta^{n}(f, \stackrel{\circ}{x})=W \theta^{n}\left({ }^{\beta} f, \stackrel{\circ}{x}\right) \in \mathscr{H}$, where ${ }^{\beta} \widetilde{f}(\vec{k})=\left(\sum_{1 \leqq i \leqq n} \vec{k}_{i}\right) \beta \widetilde{f}(\vec{k})$ for all $\vec{k} \in \mathbb{R}^{n}$, provided that ${ }^{\beta} f \in \mathscr{B}_{n}^{0}$.

iv) For all $f \in \mathscr{B}_{n}^{2},\left(1-E_{0}\right) W \theta^{n}(f, \stackrel{\circ}{x})$ is in the domain of $H$. If $\dot{x}_{i}=s$ for all $1 \leqq i \leqq n$ for some $s \in \mathbb{R}_{+}$, then for all $f \in \mathscr{B}_{n}^{4},\left(1-E_{0}\right) W \theta^{n}(f, \stackrel{\circ}{x})$ is in the domain of $H^{2}$.

v) For all $f \in \mathscr{B}_{n}^{0}, g \in \mathscr{B}_{m}^{0}$,

$$
\left(W \theta^{n}(f, \stackrel{\circ}{x}) ;\left(1-E_{0}\right) W \theta^{m}(g, \grave{y})\right)=\int_{Q} d \mu \theta^{n}(f,-\stackrel{\circ}{x})\left\{\theta^{m}(g, \stackrel{\circ}{y})-\int_{Q} d \mu \theta^{m}\left(g,,^{\circ}\right)\right\}
$$

which is a $C^{\infty}$ function of $\lambda$, and for all $v \in \mathbb{N}$,

$$
\left|\partial_{\lambda}{ }^{v}\left(W \theta^{n}(f, \stackrel{0}{x}) ;\left(1-E_{0}\right) W \theta^{m}(g, \stackrel{o}{y})\right)\right|<K \ell_{n}^{0}(f) \ell_{m}^{0}(g)
$$

for some $K \in(0, \infty)$ independent of $\lambda, f, g, \stackrel{\circ}{x}$ and $\dot{y}$.

iv) For all $\beta \in\{1,2,3\}, f \in \mathscr{B}_{n}^{\beta}, g \in \mathscr{B}_{m}^{\beta}$,

$$
\left(W \theta^{n}(f, \stackrel{\circ}{x}) ; H^{\beta} W \theta^{m}(g, \dot{y})\right)=\left.(-1)^{\beta} \partial_{\tau}^{\beta} \int_{Q} d \mu \theta^{n}(f,-\stackrel{o}{x}) \theta^{m}(g, \stackrel{\circ}{y}+\tau)\right|_{\tau=+0},
$$

where $\dot{y}+\tau=\left(\dot{y}_{1}+\tau, \ldots, \dot{y}_{m}+\tau\right)$, which is a $C^{\infty}$ function of $\lambda$, and for all $v \in \mathbb{N}$,

$$
\left|\partial_{\lambda}{ }^{v}\left(W \theta^{n}(f, \stackrel{\circ}{x}) ; H^{\beta} W \theta^{m}(g, \stackrel{\circ}{y})\right)\right|<K \ell_{n}^{\beta}(f) \ell_{m}^{\beta}(g)
$$

for some $K \in(0, \infty)$ independent of $\lambda, f, g, \stackrel{\circ}{x}$ and $\stackrel{\circ}{y}$.

vii) The statement vi) is also true for $\beta=4$ if $\dot{x}_{i}=s$ for all $1 \leqq i \leqq n$ and $\dot{y}_{j}=t$ for all $1 \leqq j \leqq m$, for some $s, t \in \mathbb{R}_{+}$.

Remark. If the interaction polynomial $P$ is even, the theorem is also true for other 
norms, still denoted by $b_{n}(f)$ and $b_{n}^{\alpha}(f)$, in the definition of which enter only the even partitions, that is those partitions $p=\left\{I_{1}, \ldots, I_{k}\right\}$ where all $\left|I_{k}\right|, I \leqq i \leqq k$, are even numbers.

The proof of the theorem (in [6]) involves the details of the construction of the weakly-coupled $P(\varphi)_{2}$-models, based on techniques such as space cut-off, uniform bounds, convergence of the cluster expansion, etc... . No use of such techniques will be found in this paper; all results presented here follow as consequences of Theorem 1.

\section{Decomposition in Zero-Time Vectors}

Let $\mathscr{D}$ be the span of $\left\{\Omega,\left(1-E_{0}\right) W \theta^{n}(f, \stackrel{\circ}{x}), n \in \mathbb{N}^{*}, f \in \mathscr{B}_{n}^{0}, \stackrel{\circ}{x} \in\left(\mathbb{R}_{+}\right)^{n}\right\}$, the set of vectors with precise Euclidean times. $\mathscr{D}$ is clearly a dense subspace of $\mathscr{H}$. The span of $\left\{\Omega,\left(1-E_{0}\right) W \theta^{n}(f, 0), n \in \mathbb{N}^{*}, f \in \mathscr{B}_{n}^{0}\right\}$, noted as $\mathscr{D}_{0}$, is the set of zero-time vectors. The zero-time Euclidean and Minkovsky fields are connected by $W \theta\left(f_{1}, 0\right) \cdots$ $\theta\left(f_{n}, 0\right)=\varphi\left(f_{1} \otimes \delta\right) \cdots \varphi\left(f_{n} \otimes \delta\right) \Omega$, and thus $\mathscr{D}_{0}$ is also the set of all polynomials of zero-time fields acting on the vacuum state.

Let $\Phi \in \mathscr{D}$ and suppose that there exists a perturbation expansion:

$$
\Phi=\sum_{i \geqq 0} \lambda^{i} \phi_{i}
$$

where each $\phi_{i} \in \mathscr{D}_{0}$. Then for all $\psi \in \mathscr{D}_{0}$ :

$$
0=\left(\psi ; \Phi-\Sigma \lambda^{i} \phi_{i}\right)=\sum_{k \geqq 0} \lambda^{k}\left[(\psi ; \Phi)_{k}-\sum_{i=0}^{k}\left(\psi ; \phi_{i}\right)_{k-i}\right],
$$

where we have expanded the scalar products, with the notation $(A, B)=\Sigma \lambda^{l}(A, B)_{l}$. Note that for fixed $k, i$ takes only a finite number of values. Thus for all $k \in \mathbb{N}$ we must have:

$$
\left(\psi ; \phi_{k}\right)_{0}=(\psi ; \Phi)_{k}-\sum_{i=0}^{k-1}\left(\psi ; \phi_{i}\right)_{k-i}
$$

Now we take $\Phi=\left(1-E_{0}\right) W \theta^{n}(f, \stackrel{\circ}{x})$ and write $\phi_{i}$ as $\left(1-E_{0}\right) \sum_{j \geqq 1} W \theta_{i}^{j}$ with $\theta_{i}^{j}=\theta^{j}\left(A_{n, i}^{j}(f, \stackrel{\circ}{x}), 0\right)$, for some functions $A_{n, i}^{j}(f, \stackrel{\circ}{x})$. If we take $\psi=\left(1-\bar{E}_{0}\right) W \theta^{r}(g, 0)$, the left-hand side of the previous formula is easily calculated (estimation of the free theory), and is $\left.\left(\psi ; W \theta_{k}^{r}\right)\right|_{\lambda=0}$. So the formula gives $\theta_{k}^{r}$ in term of the sets $\left\{\theta_{i}^{j}, j \in \mathbb{N}\right\}$ with $i<k$. The right-hand side involves the following Schwinger functions:

$$
s_{i, j}(x ; y)=\int_{\boldsymbol{Q}} d \mu: \phi\left(x_{1}\right) \cdots \phi\left(x_{i}\right):\left(: \phi\left(y_{1}\right) \cdots \phi\left(y_{j}\right):-\int_{\boldsymbol{Q}} d \mu: \phi\left(y_{1}\right) \cdots \phi\left(y_{j}\right):\right)
$$

(almost everywhere continuous functions on $\left.\mathbb{R}^{2(i+j)}\right)$. For $i=0$, “: $\phi\left(x_{1}\right) \cdots \phi\left(x_{i}\right)$ :" is replaced by 1 . We give first the formal definition of $A_{n, i}^{j}$, and verify after (Lemma 2) that it makes sense.

Definition. For all $n \in \mathbb{N}^{*}, f \in \mathscr{B}_{n}^{0}$ and $\stackrel{0}{x} \in\left(\mathbb{R}_{+}\right)^{n}$ let $A_{n, k}^{r}(f, \stackrel{\circ}{x})$ be the functions given, for all $k \in \mathbb{N}, r \in \mathbb{N}^{*}$ and $\vec{p} \in \mathbb{R}^{r}$, by: 


$$
\begin{aligned}
& \tilde{A}_{n, k}^{r}(f, \stackrel{x}{x})(\vec{p})=\frac{2^{r}}{r !} \tilde{F}(\vec{p}) \prod_{i=1}^{r} \omega_{0}\left(\vec{p}_{i}\right), \quad \text { where } F \text { is given, for all } \vec{y} \in \mathbb{R}^{r} \text {, by: } \\
& F(\vec{y})=\left.\partial_{\lambda}^{k} \frac{1}{k !} \int d^{n} \vec{x} f(\vec{x}) s p_{r, n}((\vec{y}, 0) ; x)\right|_{\lambda=0} \\
& -\left.\sum_{i=0}^{k-1} \partial_{\lambda}^{k-i} \frac{1}{(k-i) !} \sum_{j \geqq 1} \int d^{j} \vec{x} A_{n, i}^{j}(f, \stackrel{x}{x})(\vec{x}) s p_{r, j}((\vec{y}, 0) ;(\vec{x}, 0))\right|_{\lambda=0}
\end{aligned}
$$

(for $k=0$, the term containing the sum over $i$ does not appear).

An example is given in Table 1. $s p_{i, j}$ denotes the partially connected Schwinger functions (defined in Appendix 1). The connectibility property (not deduced from the above algebraic considerations) is necessary to avoid $\delta$ functions in $\tilde{A}_{n, k}^{r}(f, \stackrel{\circ}{x})$, and to have the following results.

Lemma 2. For all $n, r \in \mathbb{N}^{*}$ and $k \in \mathbb{N}, A_{n, k}^{r}: \mathscr{B}_{n}^{0} \times\left(\mathbb{R}_{+}\right)^{n} \rightarrow \mathscr{B}_{r}^{0}$. Moreover, $\ell_{r}^{0}\left(A_{n, k}^{r}(f, \stackrel{0}{x})\right)<$ $K \ell_{n}^{0}(f)$ for some $K \in(0, \infty)$ independent of $f \in \mathscr{B}_{n}^{0}$ and $\stackrel{\circ}{x} \in\left(\mathbb{R}_{+}\right)^{n}$.

This result is not trivial: it says that some Schwinger distributions can be evaluated at the functions $A_{n, k}^{r}(f, \dot{x})$, which are themselves combinations of Schwinger functions partially evaluated at $f$.

Theorem 3. For all $\Phi=\left(1-E_{0}\right) W \theta^{n}(f, \grave{x}) \in \mathscr{D}, n \geqq 1, f \in \mathscr{B}_{n}^{0}$, the following sequence of $\mathscr{D}_{0}:\left\{\phi_{i}=\left(1-E_{0}\right) \sum_{j \geqq 1} W \theta^{j}\left(A_{n, i}^{j}(f, \dot{x}), 0\right), i \in \mathbb{N}\right\}$ satisfies, for all $N \in \mathbb{N}$,

$$
\left\|\Phi-\sum_{i=0}^{N} \lambda^{i} \phi_{i}\right\|_{\mathscr{H}}<\lambda^{N+1} K b_{n}^{0}(f)
$$

for some $K \in(0, \infty)$ independent of $\lambda \in[0, \lambda], f \in \mathscr{B}_{n}^{0}$ and $\stackrel{i}{x} \in\left(\mathbb{R}_{+}\right)^{n}$.

Table 1. Decomposition in zero-time vectors of $\left(1-E_{0}\right) W \theta(f, \stackrel{\circ}{x})$

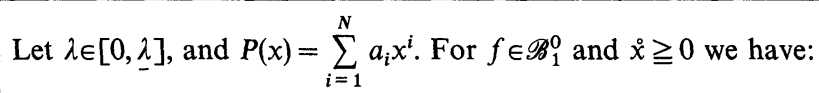

$$
\left(1-E_{0}\right) W \theta(f, \stackrel{\circ}{)})=\left(1-E_{0}\right)\left\{W \theta\left(A_{1,0}^{1}(f, \stackrel{x}{x}), 0\right)+\lambda \sum_{j=2}^{N+1} W \theta^{j}\left(A_{1,1}^{J}(f, \stackrel{x}{x}), 0\right)\right\}+O\left(\lambda^{2} \ell_{n}^{0}(f)\right)
$$

where, for all $j \geqq 1$ and $\vec{p} \in \mathbb{R}^{j}$ :

$$
\begin{aligned}
& \tilde{A}_{1,0}^{j}(f, \stackrel{\circ}{x})(\vec{p})=\delta_{j, 1} \tilde{f}\left(\vec{p}_{1}\right) \exp \left\{-\stackrel{\circ}{x} \omega_{0}\left(\vec{p}_{1}\right)\right\}, \\
& A_{1,1}^{j}(f, \stackrel{\circ}{x})=0 \text { for } j=1 \text { and for } j \geqq N+2 \text {, }
\end{aligned}
$$

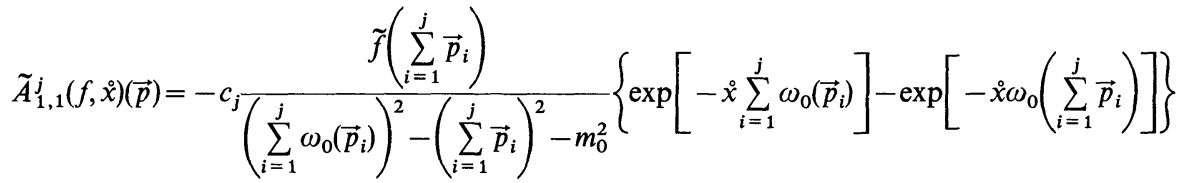

with

$$
c_{j}=\frac{(j+1) a_{j+1}}{(2 \pi)^{(j-1) / 2}}, \text { for } \quad 2 \leqq j \leqq N+1
$$




\section{Remarks on Theorem 3}

1. Nothing is said here about the growth of $K$ when $N$ increases. Thus nothing is known about the eventual convergence or resummability of the series.

2. A similar theorem exists for the decomposition in zero-time vectors of $H \Phi$ for some $\Phi \in D$ and of $H^{2} \Phi$ some $\Phi \in \mathscr{D}_{0}$.

3. When $\lambda$ varies we must suppose that $n, f$ and $\stackrel{\circ}{x}$ do not depend on $\lambda$. Note that $\mathscr{H}$ itself depends on $\lambda$ (via the construction of the measure $\mu$ ); let us denote it by $\mathscr{H}_{\lambda}$ for a moment. In the theorem $\Phi, \phi_{i} \in \mathscr{H}_{\lambda}$, so that $\lambda$ plays a double role: it indicates in which Hilbert space the vectors are, and it is a small parameter which allows perturbation expansion. The fibre bundle formalism would be better here, with base $[0, \lambda]$, fibre $\mathscr{H}_{\lambda}$, with cross sections $\Phi$ and $\phi_{i}$, supposed to be sufficiently smooth in an appropriate topology.

Proof of Lemma 2. For $k=0$, calculation of free theory gives immediately:

$$
\tilde{F}(\vec{p})=\delta_{n, r} \tilde{f}(\vec{p}) \underset{\dot{x}_{1}, \ldots, \dot{x}_{n}}{\operatorname{Sym}_{n}} \exp \left\{-\sum_{i=1}^{n}\left|\dot{x}_{i}\right| \omega_{0}\left(\vec{p}_{i}\right)\right\}
$$

where $F=A_{n, 0}^{r}(f, \stackrel{\circ}{x})$, for all $r \in \mathbb{N}^{*}$ and $\vec{p} \in \mathbb{R}^{r}$. The announced result is obvious here. Take $k \neq 0$. From its definition, $A_{n, k}^{r}(f, \dot{x})$ is a sum of functions like $T$ of Lemma 10 (in the Appendix). Thus:

$$
b_{r}^{0}\left(A_{n, k}^{r}(f, \stackrel{\circ}{x})\right)<K^{\prime} b_{n}^{0}(f)+K^{\prime \prime} \sum_{i=0}^{k-1} \sum_{j \geqq 1} b_{j}^{0}\left(A_{n, i}^{j}(f, \stackrel{\circ}{x})\right)
$$

for some $K^{\prime}, K^{\prime \prime} \in(0, \infty)$ independent of $f$ and $\stackrel{\circ}{x}$. But the sum over $j$ is finite (because $\left.\partial_{\lambda}^{k} s p_{r, j}\right|_{\lambda=0}=0$ for $j>r+k \operatorname{deg} P$ ). The conclusion follows from these considerations and from Lemma 10 by induction.

Proof of Theorem 3. It is sufficient to show that the first $N$ terms of the perturbation expansion of $\left\|\Phi-\sum_{0 \leqq i \leqq N} \lambda^{i} \phi_{i}\right\|$ vanish because of the definition of the $A_{n, k}^{r}$. In fact, by Theorem 1 and Lemma 2, all terms of the series do exist, and by the Taylor formula the remainder is automatically bounded as mentioned. This will be carried out in three steps.

1st Step. We establish that for all $\xi=W \theta^{m}(g, 0) \in \mathscr{D}_{0}$ and $N \in \mathbb{N}$, $\lim _{\lambda \rightarrow 0} \lambda^{-N}\left(\xi ; \Phi-\sum_{i=0}^{N} \lambda^{i} \phi_{i}\right)=0$. That is to say, we can pass over the connectibility properties imposed in the definition of $A_{n, k}^{r}$. Let us write $\left(\xi ; \Phi-\sum_{i=0}^{N} \lambda^{i} \phi_{i}\right)$ as:

$$
\int d^{m} \vec{y} g(\vec{y})\left[\int d^{n} \vec{x} f(\vec{x}) s_{m, n}((\vec{y}, 0) ; x)-\sum_{i=0}^{N} \lambda^{i} \sum_{j \geqq 1} \int d^{j} \vec{z} A_{n, i}^{j}(\vec{z}) s_{m, j}((\vec{y}, 0) ;(\vec{z}, 0))\right] .
$$

With Lemma 9 (in the Appendix), this becomes a sum of the following terms, involving the partially connected functions:

$$
\begin{aligned}
& \int d^{m} \vec{y} g(\vec{y}) s w_{m-k}\left(m_{m-k} \vec{y}\right) \\
& \quad \cdot\left[\int d^{n} \vec{x} f(\vec{x}) s p_{k, n}\left(\left(_{k} \vec{y}, 0\right) ; x\right)-\sum_{i=0}^{N} \lambda^{i} \sum_{j \geqq 1} \int d^{j} \vec{z} A_{n, i}^{j}(\vec{z}) s p_{k, j}\left(\left({ }_{k} \vec{y}, 0\right) ;(\vec{z}, 0)\right)\right],
\end{aligned}
$$


where $0 \leqq k \leqq m,{ }_{k} \vec{y}=\left(\vec{y}_{1}, \ldots, \vec{y}_{k}\right),_{m-k} \vec{y}=\left(\vec{y}_{k+1}, \ldots, \vec{y}_{m}\right)$. The symmetrization over $\vec{y}_{1}, \ldots, \vec{y}_{m}$ must be done. We expand in $\lambda$ the factor in brackets. The first $N$ terms vanish, because of the definition of the functions $A_{n, k}^{r}$.

$2^{\text {nd }}$ Step. We establish that for all $\xi=W \theta^{m}(g, \stackrel{\circ}{y}) \in \mathscr{D}$ and $N \in \mathbb{N}$ the following expression holds: $\lim _{\lambda \rightarrow 0} \lambda^{-N}\left(\xi ; \Phi-\sum_{i=0}^{N} \lambda^{i} \phi_{i}\right)=0$. That is to say, we can replace $\mathscr{D}_{0}$ by $\mathscr{D}$ in the statement of the $1^{\text {st }}$ step. This is the crucial point of the proof.

To see how this works, we look at the first order term:

$$
\begin{aligned}
& \left.\left(\xi ; \Phi-\sum_{i=0}^{N} \lambda^{i} \phi_{i}\right)\right|_{\lambda=0}=n ! \int\left(\prod_{i=1}^{n} \frac{d \vec{k}_{i}}{2 \omega_{0}\left(\vec{k}_{i}\right)}\right) \overline{\tilde{g}(\vec{k})} \\
& \cdot\left[\delta_{n, m} \tilde{f}(\vec{k}) \operatorname{Sym}_{\substack{\dot{x}_{1}, \ldots, \dot{x}_{n} \\
\dot{y}_{1}, \ldots, \dot{y}_{m}}} \exp \left\{-\sum_{i=1}^{n}\left|\theta \dot{y}_{i}-\dot{x}_{i}\right| \omega_{0}\left(\vec{k}_{i}\right)\right\}\right. \\
& \left.-\widetilde{F}(\vec{k}) \underset{\dot{y}_{1}, \ldots, \dot{y}_{m}}{\operatorname{Sym}} \exp \left\{-\sum_{i=1}^{n}\left|\theta \dot{y}_{i}\right| \omega_{0}\left(\vec{k}_{i}\right)\right\}\right]
\end{aligned}
$$

with $F=A_{n, 0}^{m}(f, \stackrel{\circ}{x})$, where $\theta \dot{y}_{i}=-\stackrel{\circ}{y}_{i}$. Because of the signs of $\dot{\circ}_{i}$ and $\theta \dot{y}_{j}$, all the dependence in $\dot{y}$ can be collected in a single factor giving:

$$
\left.\left(\xi ; \Phi-\sum_{i=0}^{N} \lambda^{i} \phi_{i}\right)\right|_{\lambda=0}=n ! \int\left(\prod_{i=1}^{n} \frac{d \vec{k}_{i}}{2 \omega_{0}\left(\overrightarrow{k_{i}}\right)}\right) \overline{\tilde{g}(\vec{k})} \mathscr{F}(\vec{k}, \stackrel{\circ}{x}) \underset{\dot{y}_{1}, \ldots, \dot{y}_{m}}{\operatorname{Sym} \exp }\left\{-\sum_{i=1}^{n}{\stackrel{\circ}{y_{i}}}_{i}\left(\vec{k}_{i}\right)\right\},
$$

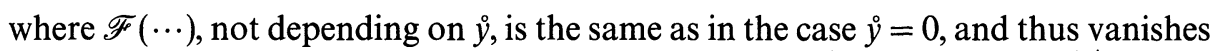
because of the property established in the first step. Thus $\left.\left(\xi ; \Phi-\sum_{0 \leqq i \leqq N} \lambda^{i} \phi_{i}\right)\right|_{\lambda=0}=0$.

We will see that at each perturbation order, the Euclidean times $\stackrel{\circ}{y}_{i}$ will appear only in a factor $\exp -\Sigma \dot{y}_{i} \omega_{0}\left(\vec{k}_{i}\right)$, multiplying an expression which vanishes because of the first step.

Let $1 \leqq v \leqq N$ and suppose (induction hypothesis) that $\lambda^{-j}\left(\xi ; \Phi-\sum_{0 \leqq i \leqq N} \lambda^{i} \phi_{i}\right) \rightarrow 0$ as $\lambda \rightarrow 0$ for all $j<v$ and all $\xi=W \theta^{m}(g, \stackrel{\circ}{y}) \in \mathscr{D}$. Let us write:

$$
I=\left.\partial_{\lambda}^{v}\left(\xi ; \Phi-\sum_{i=0}^{N} \lambda^{i} \phi_{i}\right)\right|_{\lambda=0}=\sum_{l=0}^{v} \frac{1}{l !} \int_{\mathbb{R}^{l}} d^{l} \check{z}^{\circ} F_{l}\left(\check{z}^{\circ}\right)
$$

where

$$
\begin{aligned}
F_{v}\left(\stackrel{\circ}{z}_{1}, \ldots, \stackrel{\circ}{z}_{v}\right)= & \int d^{m} \vec{y} g(\vec{y}) \int d^{n} \vec{x} \int d^{v} \vec{z} \\
& \cdot\left\{f(\vec{x}) s_{m, n}^{v}\left(\theta y ; x ; z_{1}, \ldots, z_{v}\right)-A_{n, 0}^{n}(\vec{x}) s_{m, n}^{v}\left(\theta y ;(\vec{x}, 0) ; z_{1}, \ldots, z_{v}\right)\right\}
\end{aligned}
$$

and for all $0 \leqq l<v$ :

$$
F_{l}\left(\stackrel{\circ}{z}_{1}, \ldots, \stackrel{\circ}{z}_{l}\right)=-\int d^{m} \vec{y} g(\vec{y}) \sum_{j \geqq 1} \int d^{j} \vec{x} \int d^{l} \vec{z} A_{n, v-l}^{j}(\vec{x}) s_{m j}^{l}\left(\theta y ;(\vec{x}, 0) ; z_{1}, \ldots, z_{l}\right) .
$$

We have used the following notation: $\theta y=(-\dot{y}, \vec{y}) ; " \int_{\mathbb{R}^{l}} d^{l} z^{\circ}=1$ when $l=0 . s_{m j}^{l}$ are the functions such that $\left.\partial_{\lambda}^{l} s_{m, n}(y ; x)\right|_{\lambda=0}=\int d^{2 l} z s_{m j}^{l}\left(y ; x^{\mathbb{R}} ; z\right)$ (see the derivatives of the Schwinger functions in [6]). 
With the Wick theorem the functions $s_{m j}^{l}$ can be written as follows:

$$
\begin{aligned}
& s_{m j}^{l}\left(\theta y ; x ; z_{1}, \ldots, z_{l}\right) \\
& =\sum_{\text {sym }} \sum_{r, \sigma, \tau} \int d^{m} \vec{k} f_{r, \sigma, \tau}\left(\vec{k} ; \vec{y} ; x ; z_{1}, \ldots, z_{l}\right) \exp \left\{-\sum_{i=1}^{r}\left|\theta \dot{y}_{i}-\stackrel{\circ}{x}_{\sigma(i)}\right| \omega_{0}\left(\vec{k}_{i}\right)\right\} \\
& \quad \cdot \exp \left\{-\sum_{i=r+1}^{m}\left|\theta \dot{y}_{i}-\stackrel{\circ}{z}_{\tau(i)}\right| \omega_{0}\left(\vec{k}_{i}\right)\right\}
\end{aligned}
$$

with $0 \leqq r \leqq \min \{m, j\}$ and some maps $\sigma:\{1, \ldots, r\} \rightarrow\{1, \ldots, j\}, \tau:\{r+1, \ldots, m\} \rightarrow$ $\{1, \ldots, l\}$, where the functions $f_{r, \sigma, \tau}(\cdots)$ do not depend on $\dot{y}$.

Let us suppose that only the $\stackrel{\circ}{z} \geqq 0$ contribute to $I$, that is:

$$
I=\sum_{l=0}^{v} \frac{1}{l !} \int_{0}^{\infty} d \stackrel{\circ}{z}_{1} \cdots \int_{0}^{\infty} d \dot{\circ}_{l} F_{l}\left(\stackrel{\circ}{z}_{1}, \ldots, \stackrel{\circ}{z}_{l}\right)
$$

We call this curiosity (A). If (A) holds, because of the signs of $\theta \dot{y}_{i}$ and $\dot{\circ}_{j}$, all the dependence in the variables $\dot{y}_{i}$ occurs in a factor $\exp -\sum \dot{y}_{i} \omega_{0}\left(\vec{k}_{i}\right)$, multiplying an expression which is the same as in the case $y=0$ and thus vanishes because of the first step. Then the statement of the $2^{\text {nd }}$ step is clear.

We have now to prove (A). This will follow from the induction hypothesis. Because the functions $F_{l}$ are symmetric, we can write:

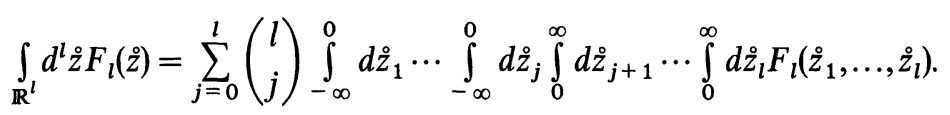

Thus $I$ becomes:

$$
I=\sum_{j=0}^{v} \frac{1}{j !} \int_{-\infty}^{0} d \stackrel{\circ}{z}_{1} \cdots \int_{-\infty}^{0} d \stackrel{\circ}{z}_{j} J_{j}\left(\stackrel{\circ}{z}_{1}, \ldots, \stackrel{\circ}{z}_{j}\right)
$$

where

$$
J_{j}\left(\stackrel{\circ}{z}_{1}, \ldots, \stackrel{\circ}{z}_{j}\right)=\sum_{l=j}^{v} \frac{1}{(l-j) !} \int_{0}^{\infty} d \check{z}_{j+1} \cdots \int_{0}^{\infty} d \check{z}_{l} F_{l}\left(\stackrel{\circ}{z}_{1}, \ldots, \stackrel{\circ}{z}_{l}\right) \quad \text { for all } \quad 0 \leqq j \leqq v .
$$

(A) holds if $I=J_{0}$, which is true if:

$$
J_{j}\left(\stackrel{\circ}{z}_{1}, \ldots, \stackrel{\circ}{z}_{j}\right)=0 \text { for all } \stackrel{\circ}{z}_{1}<0, \ldots, \stackrel{\circ}{z}_{j}<0, \quad \text { for all } j \text { with } 1 \leqq j \leqq v .
$$

We call this statement (B). Let us introduce slightly different functions $J^{\prime}$ :

$$
J_{j}^{\prime}\left(\stackrel{\circ}{z}_{1}, \ldots, \stackrel{\circ}{z}_{j}\right)=\sum_{l=j}^{v} \frac{1}{(l-j) !} \int_{\mathbb{R}^{l-j}} d \stackrel{\circ}{z}_{j+1} \cdots d \dot{\circ}_{l} F_{l}\left(\check{z}^{\circ}, \ldots, \stackrel{\circ}{z}_{l}\right) \text { for all } 0 \leqq j \leqq v .
$$

The following statement $(\mathrm{C})$ is equivalent to $(\mathrm{B})$ :

$$
J_{j}^{\prime}\left(\stackrel{\circ}{z}_{1}, \ldots, \stackrel{\circ}{z}_{j}\right)=0 \text { for all } \stackrel{\circ}{z}_{1}<0, \ldots, \stackrel{\circ}{z}_{j}<0, \text { for all } j \text { with } 1 \leqq j \leqq v .
$$

That $(B) \Rightarrow(C)$ is proved by looking at the formula:

$$
\begin{aligned}
J_{j}^{\prime}\left(\stackrel{\circ}{z}_{1}, \ldots, \stackrel{\circ}{j}_{j}\right) & =\sum_{l=j}^{v} \frac{1}{(l-j) !} \sum_{r=j}^{l}\left(\begin{array}{c}
l-j \\
r-j
\end{array}\right) \int_{-\infty}^{0} d \stackrel{\circ}{z}+1_{j} \ldots \int_{-\infty}^{0} d z_{r}^{\circ} \int_{0}^{\infty} d \stackrel{\circ}{r}_{r+1} \ldots \int_{0}^{\infty} d \stackrel{\circ}{l}_{l} F_{l}\left(\stackrel{\circ}{z}_{1}, \ldots, \stackrel{\circ}{z}_{l}\right) \\
& =\sum_{r=j}^{v} \frac{1}{(r-j) !} \int_{-\infty}^{0} d \check{z}_{j+1} \cdots \int_{-\infty}^{0} d \dot{\circ}_{r} J_{r}\left(\stackrel{\circ}{z}_{1}, \ldots, \stackrel{\circ}{z}_{r}\right) .
\end{aligned}
$$


That $(\mathrm{C}) \Rightarrow(\mathrm{B})$ is proved by induction on $j$, because $J_{v}=J_{v}^{\prime}$ and with the following expression (deduced from the preceding formula):

$$
J_{j}^{\prime}\left(\stackrel{\circ}{z}_{1}, \ldots, \stackrel{\circ}{z}_{j}\right)=J_{j}\left(\stackrel{\circ}{z}_{1}, \ldots, \stackrel{\circ}{z}_{j}\right)+\sum_{r=j+1}^{v} \frac{1}{(r-j) !} \int_{-\infty}^{0} d \stackrel{\circ}{z}_{j+1} \ldots \int_{-\infty}^{0} d \stackrel{\circ}{z}_{r} J_{r}\left(\stackrel{\circ}{z}_{1}, \ldots, \stackrel{\circ}{z}_{r}\right)
$$

for all $1 \leqq j<v$.

We prove now that $(\mathrm{C})$ actually holds. For $1 \leqq j \leqq v$ :

$$
\begin{aligned}
& J_{j}^{\prime}\left(\stackrel{\circ}{z}_{1}, \ldots, \stackrel{\circ}{z}_{j}\right) \\
& =\frac{1}{(v-j) !} \partial_{\lambda}^{v-j} \int d^{m} \vec{y} g(\vec{y}) \int d^{j} \vec{z} \int d^{n} \vec{x} f(\vec{x}) s_{m, n}^{j}\left(\theta y ; x ; z_{1}, \ldots, z_{v}\right) \\
& \quad-\sum_{l=j}^{v} \frac{1}{(l-j) !} \partial_{\lambda}^{l-j} \sum_{i \geqq 1} \int d^{m} \vec{y} g(\vec{y}) \int d^{i} \vec{x} \int d^{z} \vec{z} A_{n, v-l}^{i}(\vec{x}) S_{m, i}^{j}\left(\theta y ;(\vec{x}, 0) ;\left(\check{z}_{1}, \ldots, \stackrel{\circ}{j}_{j}\right) .\right.
\end{aligned}
$$

The functions $s_{m, i}^{j}$ can be written as integrals of sums of products of Schwinger functions (see [6]), so that we find after some simple algebra:

$$
\begin{aligned}
J_{j}^{\prime}\left(\stackrel{\circ}{z}_{1}, \ldots, \stackrel{\circ}{z}_{j}\right) & \\
= & \int d^{m} \vec{y} g(\vec{y}) \int d^{j} \vec{z} \sum_{\varnothing \subseteq K \subseteq\{1, \ldots, j\}} G_{K}\left(z_{1}, \ldots, z_{j}\right) \partial_{\lambda}^{\xi} \int_{Q} d \mu: \phi\left(\theta y_{1}\right) \cdots \phi\left(\theta y_{m}\right): \\
& \cdot\left\{\left\{\theta^{n}(f, \vec{x})-\sum_{l=j}^{v} \lambda^{v-j} \sum_{i \geqq 1} \theta_{j}^{i}\right\}\right\} \prod_{k \in K}: P\left(\phi\left(z_{k}\right)\right):\left.\right|_{\lambda=0}
\end{aligned}
$$

for some $\xi \leqq v-j$, for a.e. continuous functions $G_{K}: \mathbb{R}^{2 j} \rightarrow \mathbb{R}$ for all $K$ and with the notation $\{\{T\}\}=T-\int d \mu T$ for all $T \in L^{1}(Q, \mu)$. (Recall that all Schwinger functions are a.e. continuous functions; the integrability of the above formula with respect to the $z_{i}$ variables is assured only after having performed the $\left.\operatorname{sum} \sum_{\varnothing \subseteq K \subseteq\{1, \ldots, j\}}\right)$.

With the Wick decomposition [6, Lemma III.1.2],

$$
: \phi\left(\theta y_{1}\right) \cdots \phi\left(\theta y_{m}\right): \prod_{k}: P\left(\phi\left(z_{k}\right)\right):=\sum_{L, \alpha} h_{L, \alpha}(y, z): \prod_{i \in L} \phi\left(\theta y_{i}\right) \prod_{k} \phi\left(z_{k}\right)^{\alpha_{k}},
$$

with suitable sets $L$, maps $\alpha$ and a.e. continuous functions $h_{L, \alpha}$. We have obtained:

$$
\begin{aligned}
J_{j}^{\prime}\left(\stackrel{\circ}{z}_{1}, \ldots, \stackrel{\circ}{z}_{j}\right) & \\
= & \int d^{m} \vec{y} g(\vec{y}) \int d^{j} \vec{z} \sum_{K, L, \alpha} G_{K}(z) h_{L, \alpha}(y, z) \partial_{\lambda}^{\xi} \int_{Q} d \mu: \prod_{i \in L} \phi\left(\theta y_{i}\right) \prod_{k \in K} \phi\left(z_{k}\right)^{\alpha_{k}}: \\
& \left.\cdot\left\{\left\{\theta^{n}(f, \vec{x})-\sum_{l=j}^{v} \lambda^{v-j} \sum_{i \geqq 1} \theta_{j}^{i}\right\}\right\}\right|_{\lambda=0} .
\end{aligned}
$$

Let us suppose now that $\stackrel{\circ}{z}_{1} \leqq 0, \ldots, \stackrel{\circ}{z}_{j} \leqq 0$. Then the last integral over $d \mu$ can be written as a kernel of a scalar product of $\mathscr{H}$, and the last line of the above formula becomes:

$$
\left.\partial \sum_{\lambda}^{\xi}\left(W: \prod_{i \in L} \phi\left(y_{i}\right) \prod_{k \in K} \phi\left(\theta z_{k}\right)^{\alpha_{k}}: ; \quad \Phi-\sum_{i=0}^{v} \lambda^{i} \phi_{i}\right)\right|_{\lambda=0} .
$$


Because $\xi \leqq v-j \leqq v-1$, it follows from the induction hypothesis that this expression vanishes.

$3^{\text {rd }}$ Step. We conclude with this step. From the $2^{\text {nd }}$ step, for all $N$ and all $\xi \leqq N$ :

$$
\begin{aligned}
& \left.\partial \sum_{\lambda}^{\xi}\left(\left\|\Phi-\sum_{i=0}^{N} \lambda^{i} \phi_{i}\right\|_{\mathscr{H}}\right)^{2}\right|_{\lambda=0} \\
& \quad=\left.\partial \sum_{\lambda}^{\xi}\left(\Phi ; \Phi-\sum_{i=0}^{N} \lambda^{i} \phi_{i}\right)\right|_{\lambda=0}-\left.\sum_{j=1}^{\xi} \frac{\xi !}{(\xi-j) !} \partial_{\lambda}^{\xi-j}\left(\phi_{j} ; \Phi-\sum_{i=0}^{N} \lambda^{i} \phi_{i}\right)\right|_{\lambda=0}=0 .
\end{aligned}
$$

It follows from this and from the $2^{\text {nd }}$ step again, for all $N+1 \leqq \xi \leqq 2 N+1$ :

$$
\begin{aligned}
\left.\partial_{\lambda}^{\xi}\left(\left\|\Phi-\sum_{i=0}^{N} \lambda^{i} \phi_{i}\right\|_{\mathscr{H}}\right)^{2}\right|_{\lambda=0} & \left.\partial_{\lambda}^{\xi}\left(\left\|\Phi-\sum_{i=0}^{\xi} \lambda^{i} \phi_{i}\right\|_{\mathscr{H}}\right)^{2}\right|_{\lambda=0}+\left.\sum_{j=N+1}^{\xi} \frac{\xi !}{(\xi-j) !} \partial_{\lambda}^{\xi-j}\left(\Phi-\sum_{i=0}^{\xi} \lambda^{i} \phi_{i} ; \phi_{j}\right)\right|_{\lambda=0} \\
& +\left.\sum_{j=N+1}^{\xi} \frac{\xi !}{(\xi-j) !} \partial_{\lambda}^{\xi-j}\left(\phi_{j} ; \Phi-\sum_{i=0}^{j} \lambda^{i} \phi_{i}\right)\right|_{\lambda=0}=0 .
\end{aligned}
$$

The conclusion follows from the Taylor theorem, from Theorem 1 and then from Lemma 2.

\section{Decomposition of $\left(M^{2}-z\right)^{-1} \xi$ for $\xi \in \mathscr{D}_{0}$}

For $\Phi=\left(1-E_{0}\right) W \theta^{n}(f, 0) \in \mathscr{D}_{0}, \Phi \neq 0$, and suitable $z \in \mathbb{C}$, let us suppose that there exists a perturbation expansion such that:

$$
\Phi=\left(M^{2}-z\right) \sum_{i \geqq 0} \lambda^{i} \phi_{i}
$$

where all $\phi_{i} \in \mathscr{D}_{0}$. Then we have:

$$
\frac{1}{M^{2}-z} \Phi=\sum_{i \geqq 0} \lambda^{i} \phi_{i}
$$

We write $\phi_{i}$ as $\left(1-E_{0}\right) \sum_{j \geqq 1} W \theta_{i}^{j}$ with $\theta_{i}^{j}=\theta^{j}\left(B_{n, i}^{j}(f, z), 0\right)$ for some functions $B_{n, i}^{j}(f, z)$. Let us introduce the following notation: $d(A, z)$, for a self-adjoint operator $A$, is the smallest distance in $\mathbb{C}$ between $z$ and the spectrum of $A$, and $M_{0}$ is the operator $M$ for the free theory, that is for $\lambda=0$. We will use the following derivative of the Schwinger functions: $s p_{i, j}^{M^{2}-z}(x ; y)=\left.\left(\Delta_{s}-z\right) \operatorname{sp}_{i, j}\left(x ; y_{1}+s, \ldots, y_{j}+s\right)\right|_{s=0}$ (in the distribution sense). The definition of $B_{n, i}^{j}$, similar to that of $A_{n, i}^{j}$ (see Sect. 1), uses the following functional spaces $\mathscr{B}_{n}^{0, m}=\left\{f, f\right.$ and $\left.{ }^{2 m+2} f \in \mathscr{B}_{n}^{0}\right\}$ for all $m \in \mathbb{N}^{*}$ (for the notation ${ }^{m} f$, (see Theorem 1, iii)).

Definition. For all $k \in \mathbb{N}, n, r \in \mathbb{N}^{*}, f \in \mathscr{B}_{n}^{0, k}$ and $z \in \mathbb{C}$ with $d\left(M_{0}^{2}, z\right)>0$, let $B_{n, k}^{r}(f, z)$ be the functions given, for all $\vec{p} \in \mathbb{R}^{r}$, by:

$$
\tilde{B}_{n, k}^{r}(f, z)(\vec{p})=\frac{2 r}{r !\left(\sum_{i=1}^{r} \omega_{0}\left(\vec{p}_{i}\right)\right)^{2}-\left(\sum_{i=1}^{r} \vec{p}_{i}\right)^{2}-z}
$$


Table 2. Decomposition of $\left(M^{2}-z\right)^{-1} W \theta(f, 0)$

Let $\lambda \in[0, \lambda]$, and $P(x)=\sum_{i=1}^{N} a_{i} x^{i}$. For $f \in \mathscr{B}_{1}^{0,2}$ and $z \in \mathcal{O}$ we have:

$$
\left(1-E_{0}\right) \frac{1}{M^{2}-z} W \theta(f, 0)=\left(1-E_{0}\right) \sum_{i=0}^{1} \lambda^{i} \sum_{j \geqq 1} W \theta^{j}\left(B_{1, i}^{j}(f, z), 0\right)+O\left(\lambda^{2} \frac{d_{1,2}(z)(f)}{d\left(M^{2}, z\right)}\right)
$$

where, for all $j \geqq 1$ and $\vec{p} \in \mathbb{R}^{j}$ :

$$
\begin{gathered}
B_{1,0}^{j}(f, z)=\delta_{j, 1} \frac{1}{m_{0}{ }^{2}-z} f, \\
B_{1,1}^{1}(f, z)=-\frac{2 a_{2}}{\left(m_{0}{ }^{2}-z\right)^{2}} f, \\
\tilde{B}_{1,1}^{j}(f, z)(\vec{p})=-\frac{(j+1) a_{j+1}}{(2 \pi)^{(j-1) / 2}} \frac{1}{m_{0}^{2}-z} \frac{\tilde{f}\left(\sum_{i=1}^{j} \vec{p}_{i}\right)}{\left(\sum_{i=1}^{j} \omega_{0}\left(\vec{p}_{i}\right)\right)^{2}-\left(\sum_{i=1}^{j} \vec{p}_{i}\right)^{2}-z} \text { for } 2 \leqq j \leqq N+1, \\
B_{1,1}^{j}=0 \text { for } j \geqq N+2 .
\end{gathered}
$$

where $F$ is given, for all $\vec{y} \in \mathbb{R}^{r}$, by:

$$
\begin{aligned}
F(\vec{y})= & \left.\partial_{\lambda}^{k} \frac{1}{k !} \int d^{n} \vec{x} f(\vec{x}) s p_{r, n}((\vec{y}, 0) ; x)\right|_{\lambda=0} \\
& -\left.\sum_{i=0}^{k-1} \partial_{\lambda}^{k-i} \frac{1}{(k-i) !} \sum_{j \geqq 1} \int d^{j} \vec{x} B_{n, i}^{j}(f, z)(\vec{x}) s p_{r, j}^{M^{2}-z}((\vec{y}, 0) ;(\vec{x}, 0))\right|_{\lambda=0}
\end{aligned}
$$

(for $k=0$, the term containing the sum over $i$ does not appear).

An example is given in Table 2. Lemma 4 will show that this definition makes sense.

We introduce on $\mathscr{B}_{n}^{0, m}$ the norms $f \mapsto d_{n, m}(z)(f)$ given by:

$$
\left.d_{n, m}(z)(f)=\left(1+\frac{R}{d\left(M_{0}^{2}, z\right)}\right)^{m+1}\right) b_{n}^{0}(f)+\left(\frac{1}{d\left(M_{0}^{2}, z\right)}\right)^{m+1} b_{n}^{0}\left({ }^{2 m+2} f\right),
$$

where $R$ is some fixed arbitrary number such that $R \gg m_{0}$. We denote by $\mathcal{O}$ the following open subset of $\mathbb{C}$ :

$$
\mathcal{O}=\left\{z \in \mathbb{C},|z|<R, d\left(M^{2}, z\right)>0, d\left(M_{0}^{2}, z\right)>0\right\} .
$$

Lemma 4. For all $n, r \in \mathbb{N}^{*}$ and $k \in \mathbb{N}$ :

i) $B_{n, k}^{r}: \mathscr{B}_{n}^{0, k} \times \mathscr{O} \rightarrow \mathscr{B}_{r}^{4}$,

ii) for all $f \in \mathscr{B}_{n}^{0, k}$ the function $(z, \vec{p}) \mapsto \widetilde{B}_{n, k}^{r}(f, z)(\vec{p})$ is continuous on $\mathcal{O} \times \mathbb{R}^{r}$,

iii) for all $f \in \mathscr{B}_{n}^{0, k}$ and $z \in \mathcal{O}, b_{r}^{0}\left(B_{n, k}^{r}(f, z)\right)<K d_{n, k}(z)(f)$ and $b_{r}^{4}\left(B_{n, k}^{r}(f, z)\right)<$ $K^{\prime} d_{n, k}(z)(f)$ for some $K, K^{\prime} \in(0, \infty)$ independent of $f \in \mathscr{B}_{n}^{0, k}$ and $z \in \mathcal{O}$.

Theorem 5. For all $n, N \in \mathbb{N}, n \geqq 1, f \in \mathscr{B}_{n}^{0, N}$ and $z \in \mathcal{O}$, the following sequence of $\mathscr{D}_{0}$, $\left\{\phi_{i}=\left(1-E_{0}\right) \sum_{j \geqq 0} W \theta^{j}\left(B_{n, i}^{j}(f, z), 0\right), i \in \mathbb{N}\right\}$, satisfies:

$$
\left\|\frac{1}{M^{2}-z} \Phi-\sum_{i=0}^{N} \lambda^{i} \phi_{i}\right\|_{\mathscr{H}}<\lambda^{N+1} K \frac{d_{n, N+1}(z)(f)}{d\left(M^{2}, z\right)},
$$


where $\Phi=\left(1-E_{0}\right) W \theta^{n}(f, 0)$, for some $K \in(0, \infty)$ independent of $\lambda \in[0, \lambda], f \in \mathscr{B}_{n}^{0, N}$ and $z \in \mathcal{O}$.

\section{Remarks on Theorem 5}

1. The requirement on $f$ increases with the order $N$; but this concerns only the dependence of $f\left(\vec{x}_{1}, \ldots, \vec{x}_{n}\right)$ in the variable $\vec{x}_{1}+\cdots+\vec{x}_{n}$.

2. A similar theorem exists for the decomposition in zero-time vectors of the resolvent of $H$.

Proof of Lemma 4. We write $B_{n, i}^{j}$ instead of $B_{n, i}^{j}(f, z)$. For $k=0$, calculation of free theory gives easily:

$$
\tilde{B}_{n, 0}^{r}(\vec{p})=\delta_{n, r} \tilde{f}(\vec{p}) \frac{1}{\left(\sum_{i=1}^{r} \omega_{0}\left(\vec{p}_{i}\right)\right)^{2}-\left(\sum_{i=1}^{r} \vec{p}_{i}\right)^{2}-z}
$$

for all $r \in \mathbb{N}^{*}$ and $\vec{p} \in \mathbb{R}^{r}$. Thus ii) is obvious and $: \ell_{r}^{0}\left(B_{n, 0}^{r}\right)<\ell_{n}^{0}(f) / d\left(M_{0}^{2}, z\right)<$ $R^{-1} d_{n, 0}(z)(f)$. With the astute application of the following relation:

$$
\frac{\left(\sum_{i=1}^{r} \omega_{0}\left(\vec{p}_{i}\right)\right)^{2}}{\left(\sum_{i=1}^{r} \omega_{0}\left(\vec{p}_{i}\right)\right)^{2}-\left(\sum_{i=1}^{r} \vec{p}_{i}\right)^{2}-z}=1+\frac{\left(\sum_{i=1}^{r} \vec{p}_{i}\right)^{2}+z}{\left(\sum_{i=1}^{r} \omega_{0}\left(\vec{p}_{i}\right)\right)^{2}-\left(\sum_{i=1}^{r} \vec{p}_{i}\right)^{2}-z}
$$

we easily find:

$$
b_{r}^{4}\left(B_{n, 0}^{r}\right)<b_{r}^{0}\left({ }_{2} B_{n, 0}^{r}\right)<b_{n}^{0}(f)+\frac{b_{n}^{0}\left({ }^{2} f\right)+R b_{n}^{0}(f)}{d\left(M_{0}^{2}, z\right)}<d_{n, 0}(z)(f),
$$

where we have used the notation ${ }_{\alpha} B$ of the appendix. Take $k>1$ and let us suppose that the lemma is proved for all $B_{n, k}^{r}$, with $k^{\prime}<k$; we also suppose that $\ell_{r}^{0}\left({ }_{2} B_{n, k^{\prime}}^{r}(f, z)\right)<K^{\prime \prime} d_{n, k^{\prime}}(z)(f)$ for all $k^{\prime}<k$, for some $K^{\prime \prime} \in(0, \infty)$ independent of $f$ and $z$. Then the definition of $B_{n, k}^{r}$ gives a well defined function, satisfying ii). We define a new function $B^{\prime}$ by:

$$
\tilde{B}^{\prime}(\vec{p})=\left\{\left(\sum_{i=1}^{r} \omega_{0}\left(\vec{p}_{i}\right)\right)^{2}-\left(\sum_{i=1}^{r} \vec{p}_{i}\right)^{2}-z\right\} \tilde{B}_{n, k}^{r}(\vec{p})
$$

for all $\vec{p} \in \mathbb{R}^{r}$. From the definition of $B_{n, k}^{r}$ it follows that $B^{\prime}$ is a sum of functions like $T$ of Lemma 10 (in the appendix). Thus:

$$
\begin{aligned}
b_{r}^{0}\left(B^{\prime}\right) & <C\left\{b_{n}^{0}(f)+\sum_{0 \leqq i<k} \sum_{j \geqq 1}\left\{b_{j}^{0}\left({ }_{2} B_{n, i}^{j}\right)+b_{j}^{0}\left({ }^{2} B_{n, i}^{j}\right)+R b_{j}^{0}\left(B_{n, i}^{j}\right)\right\}\right\} \\
& <C^{\prime} d_{n, k-1}(z)(f)
\end{aligned}
$$

for some $C, C^{\prime} \in(0, \infty)$, independent of $f$ and $z$. Then:

$$
\ell_{r}^{0}\left(B_{n, k}^{r}\right)<\frac{\ell_{r}^{0}\left(B^{\prime}\right)}{d\left(M_{0}^{2}, z\right)}<R^{-1} C^{\prime} d_{n, k}(z)(f)
$$


and with the help of the previous relation $(*)$ :

$$
b_{r}^{4}\left(B_{n, k}^{r}\right)<b_{r}^{0}\left({ }_{2} B_{n, k}^{r}\right)<b_{r}^{0}\left(B^{\prime}\right)+\frac{b_{r}^{0}\left({ }^{2} B^{\prime}\right)+R b_{r}^{0}\left(B^{\prime}\right)}{d\left(M_{0}^{2}, z\right)}<C^{\prime \prime} d_{n, k}(z)(f)
$$

for some $C^{\prime \prime} \in(0, \infty)$, independent of $f$ and $z$.

Proof of Theorem 5. It is enough to prove that:

$$
\left\|\Phi-\left(M^{2}-z\right) \sum_{i=0}^{N} \lambda^{i} \phi_{i}\right\|_{\mathscr{H}}<\lambda^{N+1} K d_{n, N+1}(z)(f)
$$

for $K$ as in the theorem. As for the proof of Theorem 3, it is sufficient to show that the first $N$ terms of the perturbation expansion of $\left\|\Phi-\left(M^{2}-z\right) \sum_{0 \leqq i \leqq N} \lambda^{i} \phi_{i}\right\|$ vanish because of the definition of the $B_{n, k}^{r}$. By Theorem 1 and Lemma 4, all terms of the series exist (all $\phi_{i}$ are in the domain of $H^{2}$ which contains the domain of $M^{2}$ ) and by the Taylor formula the remainder is automatically bounded as mentioned. The end of the proof is exactly the same as for Theorem 3, and all arguments can be repeated.

\section{Zero-Time One-Particle States}

We use now a new piece of information. In [9] it is shown that the spectrum of the mass operator $M$ is contained in the following subset of $\mathbb{R}_{+}$:

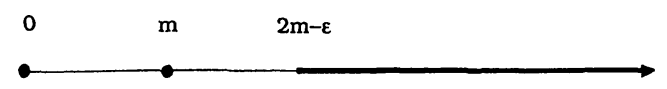

where 0 and $m$ are eigenvalues, and $m$ and $\varepsilon$ are continuous functions of $\lambda \in[0, \lambda]$ such that $m \rightarrow m_{0}$ and $\varepsilon \rightarrow+0$ as $\lambda \rightarrow 0$. The subspace corresponding to 0 is the vacuum $E_{0} \mathscr{H}=\{c \Omega, c \in \mathbb{C}\}$. The subspace corresponding to $m$, which carries an irreducible representation of the Poincare group, is the set of one-particle states, denoted as $E_{m} \mathscr{H}$.

Thus for $\lambda$ sufficiently small, there exists a circle $\mathscr{C}$ in $\mathbb{C}$, with center $m^{2}$, such that $d\left(M_{0}^{2}, z\right)>\frac{1}{4} m_{0}^{2}$ and $d\left(M^{2}, z\right)>\frac{1}{4} m_{0}^{2}$ for all $z \in \mathscr{C}$ (see the figure just below). Let us call $\lambda$ the maximal value in $(0, \lambda]$ for which these conditions hold for all $\lambda \in[0, \lambda]$ We fix now $\lambda \in[0, \lambda]$. We fix now $\lambda \in[0, \lambda]$ for the rest of this paper. The projector $E_{m}$ can now be written as:

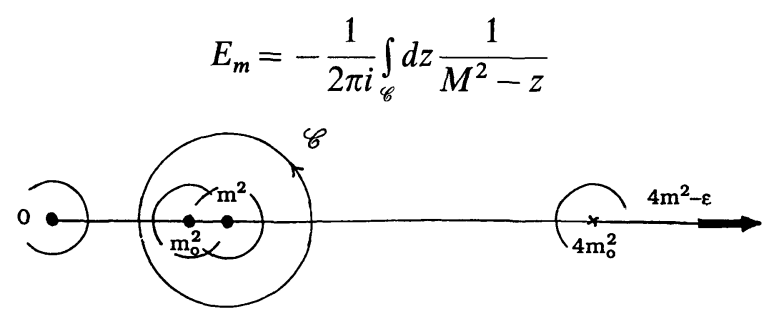

Definition. The $N^{\text {th }}$ approximations of zero-time one-particle states, with $N \in \mathbb{N}$, are defined by:

$$
\begin{aligned}
& \Psi_{N}(f)=\left(1-E_{0}\right) W \Theta_{N}(f), \\
& \Theta_{N}(f)=\sum_{i=0}^{N} \lambda^{i} \sum_{j \geqq 1} \theta^{j}\left(D_{i}^{j}(f), 0\right),
\end{aligned}
$$


Table 3. First approximation of zero-time one-particle states $\Psi_{1}(f)$

Let $\lambda \in[0, \lambda]$, and $P(x)=\sum_{i=1}^{N} a_{i} x^{i}$. For all $f \in \mathscr{B}_{1}^{4}$ we have:

$$
\Psi_{1}(f)=\left(1-E_{0}\right)\left\{W \theta\left(D_{0}^{1}(f), 0\right)+\lambda \sum_{j=2}^{N+1} W \theta^{j}\left(D_{1}^{j}(f), 0\right)\right\}+O\left(\lambda^{2} b_{1}^{4}(f)\right)
$$

where, for all $j \geqq 1$ and $\vec{p} \in \mathbb{R}^{j}$ :

$$
\begin{gathered}
D_{0}^{j}(f)=\delta_{j, 1} f \\
D_{1}^{j}(f)=0 \text { for } j=1 \text { and for } j \geqq N+2, \\
\tilde{D_{1}^{j}(f)(\vec{p})=-\frac{(j+1) a_{j+1}}{(2 \pi)^{(j-1) / 2}}\left(\sum_{i=1}^{j} \vec{p}_{i}\right)} \text { for all } 2 \leqq j \leqq N+1 .
\end{gathered}
$$

for all $f \in \mathscr{B}_{1}^{0, N}$, where for all $i \in\{1, \ldots, N\}, j \in \mathbb{N}^{*}$ and $\vec{p} \in \mathbb{R}^{j}$ :

$$
\tilde{D}_{i}^{j}(f)(\vec{p})=-\frac{1}{2 \pi i} \int_{\mathscr{C}} d z \tilde{B}_{1, i}^{j}(f, z)(\vec{p}) .
$$

See Table 3 for explicit calculations. From Theorem 5 and from the knowledge of the spectrum of $M$ pointed out above, we deduce the following properties of $D_{i}^{j}(f)$.

Lemma 6. For all $r \in \mathbb{N}^{*}$ and $k \in \mathbb{N}, D_{k}^{r}: \mathscr{B}_{1}^{4(k+1)} \rightarrow \mathscr{B}_{r}^{4}$. Moreover, $b_{r}^{4}\left(D_{k}^{r}(f)\right)<$ $K \ell_{1}^{4(k+1)}(f)$ for some $K \in(0, \infty)$ independent of $f \in \mathscr{B}_{1}^{4(k+1)}$.

We collect now the properties of $\Psi_{N}(f)$.

Theorem 7. For all $N \in \mathbb{N}$ and $f, g \in \mathscr{B}_{1}^{4(N+1)}$ :

i) $\Psi_{N}(f) \in\left(1-E_{0}\right) \mathscr{H}$ and $\Psi_{N}(f)$ belongs to the domain of $H^{2}$,

ii) $\left\|\left(1-E_{m}\right) \Psi_{N}(f)\right\|_{\mathscr{H}}=O\left(\lambda^{N+1} b_{1}^{4(N+1)}(f)\right)$,

iii) $\vec{P} \Psi_{N}(f)=\Psi_{N}\left({ }^{1} f\right)$

$$
H^{2} \Psi_{N}(f)=\Psi_{N}\left({ }_{2} f\right)+O\left(\lambda^{N+1} b_{1}^{4(N+1)}(f)\right),
$$

iv) $\left(\Psi_{N}(f) ; \Psi_{N}(g)\right)=C \int_{\mathbb{R}} \frac{d \vec{k}}{2 \omega(\vec{k})} \bar{f}(\vec{k}) \tilde{g}(\vec{k})+O\left(\lambda^{N+1} b_{1}^{4(N+1)}(f) \ell_{1}^{4(N+1)}(g)\right) \quad$ for

v) Let us suppose that $f \neq 0$. There exist $K, K^{\prime} \in(0, \infty)$, depending only on $m_{0}$ and $P$, such that, if $\lambda<1 / K^{\prime}$, then:

$$
\frac{\left(\Psi_{N}(f) ; M^{2} \Psi_{N}(f)\right)}{\left\|\Psi_{N}(f)\right\|_{\mathscr{H}}^{2}}=m^{2}+\lambda^{2 N+2} \mathscr{R}_{N}(\lambda, f)
$$

where $\left|\mathscr{R}_{N}(\lambda, f)\right|<\frac{K}{1-\lambda K^{\prime}} \frac{b_{1}^{4(N+1)}(f)}{b_{1}^{0}(f)}$.

Notation. We have used the function $\omega(\vec{k})=\sqrt{\vec{k}^{2}+m^{2}}$ and the following notation: ${ }_{\alpha} f$ is the function deduced from $f$ by ${ }_{\alpha} \tilde{f}(\vec{k})=\omega(\overrightarrow{\mathrm{k}})^{\alpha} \widetilde{f}(\vec{k})$, and $O(F(\lambda, f, g))$ is an 
expression with absolute value bounded by $F(\lambda, f, g)$ times a constant independent of $\lambda, f$ and $g$, where $F$ is a positive function and $\lambda \in[O, \lambda], f$ and $g \in \mathscr{B}_{1}^{4(N+1)}$.

\section{Remark on the Theorem}

1. In the theorem, $m$ is the mass of one particle with interaction, depending on $\lambda$.

2. To understand the sense of ii), we note that, by iv), the $\left\|\Psi_{N}(f)\right\|_{\mathscr{H}}$ do not vanish in general, as $\lambda \rightarrow 0$.

3. v) states that the Rayleigh quotient is advantageous: it gives the eigenvalue up to $O\left(\lambda^{2 N+2}\right)$ when the eigenvector is known only up to $O\left(\lambda^{N+1}\right)$, but this requires a limitation of the values of $\lambda$.

Proof of Lemma 6. Because $\lambda<\lambda$ (thus $\mathscr{C} \subset \mathcal{O}$ ) and by Lemma 4, ii) the function $\vec{p} \mapsto \widetilde{D}_{i}^{j}(f)(\vec{p})$ is continuous. From the Cauchy-Schwartz inequality, we find:

$$
\left|\tilde{D}_{i}^{j}(f)(\vec{p})\right|^{2}=\left|\frac{1}{2 \pi i} \int_{\mathscr{C}} d z \tilde{B}_{1, i}^{j}(f, z)(\vec{p})\right|^{2}<K^{\prime} \int_{\mathscr{C}} d z\left|\tilde{B}_{1, i}^{j}(f, z)(\vec{p})\right|^{2}
$$

for $K^{\prime}=($ length of $\mathscr{C}) / 2 \pi$. From Lemma 4 we obtain:

$$
\begin{aligned}
\ell_{r}^{4}\left(D_{k}^{r}(f)\right)^{2} & <K^{\prime} \int_{\mathscr{C}} d z b_{r}^{4}\left(B_{1, k}^{r}(f, z)\right)^{2} \\
& <K^{\prime \prime} b_{1}^{0}(f)^{2}+K^{\prime \prime \prime} b_{1}^{0}\left(\left(^{2 k+2} f\right)^{2}<K^{2} b_{1}^{4(k+1)}(f)^{2}\right.
\end{aligned}
$$

for some $K, K^{\prime \prime}, K^{\prime \prime \prime} \in(0, \infty)$ independent of $f$.

Proof of Theorem 7. i) Follows from Theorem 1 and Lemma 6.

ii) We recall that from Theorem 5:

$$
\frac{1}{M^{2}-z} \Phi=\sum_{i=0}^{N} \lambda^{i} \phi_{i}-\lambda^{N+1} \mathscr{K}_{N+1}(f, z)
$$

where $\Phi=\left(1-E_{0}\right) W \theta^{1}(f, 0)$ and $\phi_{i}=\left(1-E_{0}\right) \sum_{j \geqq 0} W \theta^{j}\left(B_{1, i}^{j}(f, z), 0\right)$ for all $N \in \mathbb{N}$, $i \in\{0, \ldots, N\}, f \in \mathscr{B}_{n}^{0, N}$ and $z \in \mathcal{O} . \mathscr{K}_{N+1}(f, z)$ is a vector of $\mathscr{H}$ bounded as given in Theorem 5 . We integrate over $z \in \mathscr{C}$ and divide by $2 \pi i$ to find:

$$
E_{m} \Phi=\Psi_{N}(f)+\lambda^{N+1} \frac{1}{2 \pi i} \int_{\mathscr{C}} d z \mathscr{K}_{N+1}(f, z) .
$$

We obtain the result by applying $\left(1-E_{m}\right)$ to both sides (the term with $\mathscr{K}$ can be controlled using the same methods as in the proof of Lemma 6).

iii) The first formula holds for all zero-time vectors with suitable $f$. For the second we apply $M^{2}=H^{2}-\vec{P}^{2}$ to both sides of $(* *)$, which gives:

$$
H^{2} \Psi_{N}(f)=\left(m^{2}+\vec{P}^{2}\right) \Psi_{N}(f)+\lambda^{N+1}\left(m^{2}-M^{2}\right) \frac{1}{2 \pi i} \int_{\mathscr{C}} d z \mathscr{K}_{N+1}(f, z) .
$$

Note that the methods of the proof of Lemma 6 can show that the term with $\mathscr{K}$ exists, i.e. $\int \mathscr{K}$ is in the domain of $M^{2} \subset H^{2}$.

iv) Let us denote by $\Phi(f)$ the left-hand side of relation (**). Because $E_{m} \mathscr{H}$ carries 
an irreductible representation of the Poincaré group, we must have:

$$
(\Phi(f) ; \Phi(g))=C \int_{\mathbb{R}} \frac{d \vec{k}}{2 \omega(\vec{k})} \bar{f}(\vec{k}) \tilde{g}(\vec{k}),
$$

where $C$ is some constant (which can depend on $\lambda$ ). The announced relation follows from the formula $(* *)$.

v) We use (**) and that $E_{m} \Phi$ is an eigenvector of $M^{2}$ to obtain:

$$
\begin{aligned}
\left(\Psi_{N}(f) ; M^{2} \Psi_{N}(f)\right) & =\left(E_{m} \Phi-\lambda^{N+1} K ; M^{2}\left(E_{m} \Phi-\lambda^{N+1} K\right)\right) \\
& =m^{2}\left\|E_{m} \Phi\right\|^{2}-2 m^{2} \lambda^{N+1} \mathscr{R} e\left(\Psi_{N}(f) ; K\right)+\lambda^{2(N+1)}\left(K ; M^{2} K\right) \\
& =m^{2}\left\|\Psi_{N}(f)\right\|^{2}+\lambda^{2(N+1)}\left(K ;\left(M^{2}-m^{2}\right) K\right),
\end{aligned}
$$

where $K=(2 \pi i)^{-1} \int_{\mathscr{C}} d z \mathscr{K}_{N+1}(f, z)$. Putting this into the Rayleigh quotient gives the announced formula, with $\mathscr{R}_{N}(\lambda, f)=\frac{\left(K ;\left(M^{2}-m^{2}\right) K\right.}{\left\|\Psi_{N}(f)\right\|^{2}}$. The numerator is bounded by a constant time $b_{1}^{4(N+1)}(f)^{2}$. For the denominator we use the Taylor formula: $\left\|\Psi_{N}(f)\right\|^{2}=\left.\left\|\Psi_{N}(f)\right\|^{2}\right|_{\lambda=0}+\lambda\left(\frac{\partial}{\partial \lambda}\left\|\Psi_{N}(f)\right\|^{2}\right)_{\lambda=\xi}$ for some $0<\xi<\lambda$. Thus $\left\|\Psi_{N}(f)\right\|^{2}>\frac{1}{2} b_{1}^{0}(f)-\lambda K^{\prime \prime} b_{1}^{0}(f)$ for some $K^{\prime \prime} \in(0, \infty)$ by Theorem 1 .

\section{Some Interesting Vectors}

For all $N$ and $n \in \mathbb{N}, n \geqq 2$, let us consider the following vectors:

$$
\begin{aligned}
& \Xi_{N}^{n}(f)=\left(1-E_{0}-E_{m}\right) W \Theta_{N}^{n}(f), \\
& \Theta_{N}^{n}(f)=\Theta_{N}\left(f_{1}\right) \cdots \Theta_{N}\left(f_{n}\right)
\end{aligned}
$$

for $f \in\left(\mathscr{B}_{1}^{4 N+4}\right)^{n}$. With the above methods these can be written as:

$$
\Xi_{N}^{n}(f)=\left(1-E_{0}\right) \sum_{i=0}^{N} \lambda^{i} \sum_{j \geqq 1} W \theta^{j}\left(E_{n, i}^{j}(f), 0\right)+O\left(\lambda^{N+1}\right),
$$

where the functions $E_{n, i}^{j}(f)$ can be written explicitly. We now define new vectors $\Psi_{N}^{n}(f)$ by taking only the first part of the right-hand side of the previous formula:

$$
\Psi_{N}^{n}(f)=\left(1-E_{0}\right) \sum_{i=0}^{N} \lambda^{i} \sum_{j \geqq 1} W \theta^{j}\left(E_{n, i}^{j}(f), 0\right) .
$$

Such vectors will be used in [3] to construct ansatz approaching the $n$ particles states at low energy. It follows from the previous results that they immediately satisfy:

Proposition 8. For all $n, N \in \mathbb{N}$ and $f \in\left(\mathscr{B}_{1}^{4 N+4}\right)^{n}$ :

i) $\Psi_{N}^{n}(f) \in\left(1-E_{0}\right) \mathscr{H}$ and belongs to the domain of $\mathrm{H}^{2}$,

ii) $\left\|\left(1-E_{m}\right) \Psi_{N}^{n}(f)\right\|_{\mathscr{H}}=O\left(\lambda^{N+1} \prod_{i=1}^{n} b_{1}^{4(N+1)}\left(f_{i}\right)\right)$,

iii) $\vec{P} \Psi_{N}^{n}(f)=\Psi_{N}^{n}\left({ }^{1} f\right)$. 
To understand the sense of ii), we note that the $\left\|\Psi_{N}^{n}(f)\right\|_{\mathscr{H}}$ do not vanish in general, as $\lambda \rightarrow 0$.

\section{Appendix. The Partially Connected Schwinger Functions}

The Schwinger functions $s_{i, j}$ defined in Sect. 1 can be written as a sum of products over the partitions of $\{1, \ldots, i+j\}$ (see [6]); the partially connected Schwinger functions $s p_{i, j}$ are those obtained when the sum runs only over the partitions connected with respect to $\{1, \ldots, i\}$ (defined in $[6$, Appendix B]). That is:

$$
s p_{i, j}(x ; y)=\sum_{p \in \mathscr{P}_{I^{\prime}(I \cup J)}} \prod_{I^{\prime} \cup J^{\prime} \in p, I^{\prime} \subset I, J^{\prime} \subset J} f_{I^{\prime}, J^{\prime}}\left(x_{I}, y_{J^{\prime}}\right),
$$

where $I=\{1, \ldots, i\}, J=\{i+1, \ldots, i+j\}, x_{I^{\prime}}=\left\{x_{i}, i \in I^{\prime}\right\}$ and $y_{J^{\prime}}=\left\{y_{j}, j \in J^{\prime}\right\}$. The symmetric functions $f_{K, L}$ are given by:

$$
f_{K, L}(u, v)=s w t_{m}(u, v)-\delta_{1,|K|} \delta_{1,|L|} c(u-v)
$$

for all $K, L \subset \mathbb{N}, m=|K|+|L|$. The functions $s w t_{m}$ and $c$ are given in [6].

Lemma 9. For all $i, j \in N, j \geqq 1$, and all $x \in \mathbb{R}^{2 i}, y \in \mathbb{R}^{2 j}$ :

$$
s_{i, j}(x ; y)=\sum_{I^{\prime} \subset I} s w_{\left|I^{\prime}\right|}\left(x_{I^{\prime}}\right) s p_{\left|I-I^{\prime}\right|, j}\left(x_{I-I^{\prime}} ; y\right),
$$

where $I=\{1, \ldots, i\}$.

Proof. This follows from the decomposition of $\mathscr{P}(I \cup J)$ into connected partitions (see [6, Appendix B, Lemma B10]), and from the definition of the Schwinger functions $s w_{i}, s w t_{i}, s_{i}, s p_{i, j}$.

For all $n, r \in \mathbb{N}^{*}, f \in \mathscr{B}_{n}^{0}, \stackrel{\circ}{x} \in\left(\mathbb{R}_{+}\right)^{n}$ and $\left.\stackrel{\circ}{\dot{y} \in\left(\mathbb{R}_{+}\right.}\right)^{r}$, let us define the functions:

$$
T_{r}^{\alpha}(y)=\left.\partial_{s}^{\alpha} \int d^{n} \vec{x} f(\vec{x}) s p_{r, n}(y ;(\vec{x}, \stackrel{\circ}{x}+s))\right|_{s=+0}
$$

for all $\alpha \in \mathbb{N}$ and $\vec{y} \in \mathbb{R}^{r}$ (where $\dot{x}+s=\left(\dot{\circ}_{1}+s, \ldots, \dot{\circ}_{n}+s\right)$ ). We denote by $\tilde{T}_{r}^{\alpha}(\vec{p})$ the Fourier transform of $\vec{y} \mapsto T_{r}^{\alpha}(y)$ (all other variables being fixed). We will use the notation ${ }_{\alpha} \tilde{f}(\vec{p})=\left(\prod_{i=1}^{n} \omega_{0}\left(\vec{p}_{i}\right)\right)^{\alpha} \tilde{f}(\vec{p})$ for all $\alpha \in \mathbb{N}, \vec{p} \in \mathbb{R}^{n}$ and suitable function $f: \mathbb{R}^{n} \rightarrow \mathbb{C}$ (do not confuse with the notation of Sect. 3, which uses $\omega$ instead of $\omega_{0}$ ).

Lemma 10. Fix $n, r \in \mathbb{N}^{*}, \stackrel{\circ}{x} \in\left(\mathbb{R}_{+}\right)^{n}$ and $\stackrel{\circ}{y} \in\left(\mathbb{R}_{+}\right)^{r}$. For all $v, \alpha \in \mathbb{N}$ with $\alpha \leqq 2$ and ${ }_{\alpha} f \in \mathscr{B}_{n}^{0}$, the function

$$
\tilde{T}: \vec{p} \mapsto\left(\prod_{i=1}^{r} \omega_{0}\left(\vec{p}_{i}\right)\right) \partial_{\lambda}^{v} \widetilde{T}_{r}^{\alpha}(\vec{p})
$$

satisfies: $T \in \mathscr{B}_{r}^{0}$, and $b_{r}^{0}(T)<K b_{n}^{0}\left({ }_{\alpha} f\right)$ for some $K \in(0, \infty)$ independent of $\lambda, f, \stackrel{\circ}{x}$ and $\stackrel{\circ}{y}$.

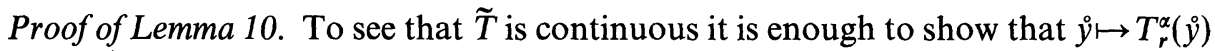
is in $L^{1}\left(\mathbb{R}^{r}\right)$ (the other variables being fixed). This is true because the truncated functions like $f_{K, L}$ admit bounds which contain an exponential and products of logarithms [6, 1st Part, Lemma, Sect. IV]. Because of the summation on connected partitions only, the functions $s p_{i j}$ and then $T_{r}^{\alpha}(y)$ admit such an exponential bound. They are in $L^{1}$. From the WTI Programme [6], $\widetilde{T}\left(\vec{k}_{1}, \ldots, \vec{k}_{r}\right)$ is a sum of the 
following terms:

$$
\int\left(\prod_{i=1}^{n-l} \frac{d \vec{p}_{i}}{2 \omega_{0}\left(\vec{p}_{i}\right)}\right) \tilde{f}\left(\vec{k}_{1}, \ldots, \vec{k}_{l}, \vec{p}_{1}, \ldots, \vec{p}_{n-l}\right) h(\vec{p}, \vec{k}, \stackrel{\circ}{x}, \stackrel{\circ}{y}) \prod_{I \cup J \in p} \delta\left(\sum_{i \in I} \vec{k}_{j}+\sum_{j \in J} \vec{p}_{j}\right),
$$

where

$$
\begin{aligned}
h(\vec{p}, \vec{k}, \dot{\circ}, \dot{y})= & C_{l} \partial_{s}^{\alpha} \int\left(\prod_{i=1}^{n-l} \frac{\omega_{0}\left(\vec{p}_{i}\right) d \dot{\circ}_{i}}{\dot{p}_{i}^{2}+\omega_{0}\left(\vec{p}_{i}\right)^{2}}\right)\left(\prod_{i=l+1}^{r} \frac{\omega_{0}\left(\vec{k}_{i}\right) d \dot{k}_{i}}{{\stackrel{\circ}{k_{i}}}^{2}+\omega_{0}\left(\vec{k}_{i}\right)^{2}}\right) \exp \chi(\stackrel{\circ}{x}+s, \stackrel{\circ}{y}, k, p) \\
& \left.\cdot \partial_{\lambda}^{v} \prod_{I \cup J \in p} \delta\left(\sum_{i \in I}{\stackrel{\circ}{k_{j}}}_{j}+\sum_{j \in J}{\stackrel{\circ}{p_{j}}}_{j}\right) \Sigma_{\lambda}^{|I \cup J|}\left(\left\{k_{i}, p_{j}, i \in I, j \in J\right\}\right)\right|_{s=0}
\end{aligned}
$$

with

$$
\chi(\stackrel{\circ}{x}+s, \dot{y}, k, p)=-\sum_{i=1}^{l}\left|\theta \dot{y}_{i}-\dot{\circ}_{i}-s\right| \omega_{0}\left(\vec{k}_{i}\right)+i \sum_{j=1}^{n-l} \dot{p}_{j}\left(\stackrel{\circ}{i}_{i}+s\right)+i \sum_{j=l+1}^{r}{\stackrel{\circ}{k_{j}} \dot{y}_{i}},
$$

where $0 \leqq l \leqq \min \{r, j\}, C_{l}$ is a combinatorial factor, $p$ is a partition of $I^{\prime} \cup J^{\prime}$ with $I^{\prime} \approx\{l+1, \ldots, r\}$ and $J^{\prime} \approx\{1, \ldots, n-l\}$, which is connected with respect to $I^{\prime}$ (that is to say, all $\delta$ distributions have at least one $\vec{p}_{i}$ or one $\dot{p}_{j}$ variable; thus all $\delta$ can disappear after some trivial integrations, to give an ordinary Lebesgue integral). An overall summation for symmetrization on $\vec{k}_{1}, \ldots, \vec{k}_{n}$, on $\dot{x}_{1}, \ldots, \dot{x}_{n}$ and on $\dot{y}_{1}, \ldots, \dot{y}_{r}$ has to be done. The functions $\Sigma_{\lambda}^{i}$ are given and discussed in [6]. It is proved in $[6,2$ nd Part $]$ that $h$ satisfies:

$$
|h(\vec{p}, \vec{k}, \stackrel{\circ}{x}, \stackrel{\circ}{y})|<C^{\prime}\left(\sum_{i=1}^{n-l} \omega_{0}\left(\vec{p}_{i}\right)+\sum_{i=l+1}^{r} \omega_{0}\left(\vec{k}_{i}\right)\right)^{\alpha}
$$

for some constant $C^{\prime}{ }_{l} \in(0, \infty)$, independent of $\stackrel{\circ}{x}, \stackrel{\circ}{y}$ and $\lambda$. Then $\widetilde{T}(\vec{k})$ is bounded by a sum of following terms:

$$
K^{\prime} \int\left(\prod_{i=1}^{n-l} \frac{d \vec{p}_{i}}{\omega_{0}\left(\vec{p}_{i}\right)}\right)\left|\alpha \tilde{f}\left(\vec{k}_{1}, \ldots, \vec{k}_{l}, \vec{p}_{1}, \ldots, \vec{p}_{n-l}\right)\right| \prod_{I \cup J \in p} \delta^{(1)}\left(\sum_{i \in I} \vec{k}_{j}+\sum_{j \in J} \vec{p}_{j}\right)
$$

for some $K^{\prime} \in(0, \infty)$. Thus by the Cauchy-Schwartz inequality:

$$
|\tilde{T}(\vec{k})|<K^{\prime \prime}\left[\left.\left.\sum_{l} \sum_{p} \sum_{\text {sym }} \int\left(\prod_{i=1}^{n-l} \frac{d \vec{p}_{i}}{\omega_{0}\left(\vec{p}_{i}\right)}\right)\right|_{\alpha} \tilde{f}(\cdots)\right|^{2} \prod_{I \cup J \in p} \delta\left(\sum \vec{k}_{j}+\sum \vec{p}_{j}\right)\right]^{1 / 2}
$$

for some $K^{\prime \prime} \in(0, \infty)$. We put this inequality in the definition of $\ell_{r}^{0}(T)$, which become bounded by a sum of the following terms:

$$
\begin{aligned}
& \left.\left.K^{\prime \prime} \int\left(\prod_{i=1}^{r} \frac{d \vec{k}_{i}}{\omega_{0}\left(\vec{k}_{i}\right)}\right)\left(\prod_{K \in p^{\prime}} \delta\left(\sum_{i \in K} \vec{k}\right)\right) \int\left(\prod_{i=1}^{n-l} \frac{d \vec{p}_{i}}{\omega_{0}\left(\vec{p}_{i}\right)}\right)\right|_{\alpha} \tilde{f}\left(\vec{k}_{1}, \ldots, \vec{k}_{l}, \vec{p}_{1}, \ldots, \vec{p}_{n-l}\right)\right|^{2} \\
& \cdot \sum_{I \cup J \in p} \delta\left(\sum_{i \in I} \vec{k}_{j}+\sum_{j \in J} \vec{p}\right),
\end{aligned}
$$

where $p^{\prime}$ is a partition of $\{1, \ldots, r\}$. Note that because of the imposed connectibility properties, all $\delta$ distributions have different arguments. The integration over the variables $\vec{k}_{l+1}, \ldots, \vec{k}_{r}$ (not appearing in $f(\cdots)$ ) is easily controlled. The result follows from some change of variables. 


\section{References}

1. Bros, J., Iagolnitzer, D.: 2-particle asymptotic completeness and bound states in weakly coupled quantum field theories. Commun. Math. Phys. 119, 331-351 (1988)

2. Frochaux, E.: A variational proof of the existence of a bound state in a relativistic quantum model with weak coupling. Helv. Phys. Acta 61, 923-957 (1988)

3. Frochaux, E.: The bound states of the weakly-coupled $P(\varphi)_{2}$ models obtained by the variational perturbation method. In preparation

4. Frochaux, E.: Relativistic corrections to the Schrödinger equation obtained by the variational perturbation method applied to the weakly-coupled $P(\varphi)_{2}$ models. In preparation

5. Frochaux, E.: Probability axioms for quantum field theory. Helv. Phys. Acta 62, 1038-1069 (1989). See also [7]

6. Frochaux, E.: Sur le domaine de l'hamiltonien dans les modèles $P(\varphi)_{2}$. Thèse de l'Université de Lausanne, Suisse (1988). On the domain of the Hamiltonian in weakly coupled $P(\varphi)_{2}$ models, In preparation. See also [7]

7. Frochaux, E.: Some analytic results about weakly-coupled $P(\varphi)_{2}$ models. Rapport interne du Département de Mathématique, Ecole Polytechnique Fédérale de Lausanne, Suisse. In preparation. The bound-states in Quantum Field Theory: review of some analytic problems of the variational perturbation method (submitted for publication)

8. Glimm, J., Jaffe, A. Spencer, T.: Physics of quantum field models. In: Velo, G., Wightman, A. (eds.) Constructive Quantum Field Theory. Proceedings, Eric 1973. Lecture Notes in Physics, Vol. 25, pp. 132-198. Berlin, Heidelberg, New York: Springer 1973

9. Glimm, J., Jaffe, A., Spencer, T.: The Wightman axioms and particle structure in the $P(\varphi)_{2}$ quantum field model. Ann. Math. 100, 585-632 (1974). See also: Glimm, J., Jaffe, A.: Quantum Physics, Second Edition. Berlin, Heidelberg, New York: Springer 1987

Communicated by A. Jaffe 
